\title{
Philosophiques
}

\section{Spinoza et le problème juif de la théodicée}

\section{Steven Nadler}

Volume 29, numéro 1, printemps 2002

Spinoza sous le prisme de son anthropologie

URI : https://id.erudit.org/iderudit/009562ar

DOI : https://doi.org/10.7202/009562ar

Aller au sommaire du numéro

Éditeur(s)

Société de philosophie du Québec

ISSN

0316-2923 (imprimé)

1492-1391 (numérique)

Découvrir la revue

Citer cet article

Nadler, S. (2002). Spinoza et le problème juif de la théodicée. Philosophiques, 29(1), 41-56. https://doi.org/10.7202/009562ar d'utilisation que vous pouvez consulter en ligne.

https://apropos.erudit.org/fr/usagers/politique-dutilisation/ 


\title{
Spinoza et le problème juif de la théodicée
}

\author{
STEVEN NADLER \\ University of Wisconsin, Madison \\ smnadler@facstaff.wisc.edu
}

\begin{abstract}
RÉSUMÉ. - On considère souvent qu'il n'y a pas de place dans la philosophie de Spinoza pour le problème de la théodicée ou pour la question de la conciliation de la justice, de la sagesse et de la puissance divines avec la présence apparente de mal et d'imperfection dans le monde. Son rejet d'un Dieu providentiel semblerait écarter à soi seul toute approche spinoziste du problème. Toutefois, Spinoza a bel et bien beaucoup à dire sur la question de la théodicée. En niant l'immortalité personnelle de l'âme, Spinoza offre de manière, je crois, pleinement consciente une réponse à l'approche traditionnelle qu'on trouve au sein de la philosophie juive médiévale, celle qui repose sur les récompenses à attendre de la vertu dans le «monde à venir ». De plus, on trouve effectivement les composantes de l'approche de Spinoza dans cette tradition antérieure, et la conception de Spinoza est une continuation naturelle d'un courant à l'intérieur du rationalisme juif médiéval.
\end{abstract}

\begin{abstract}
It is often assumed that there is no room in Spinoza's philosophy for dealing with the theodicy problem, or the question of how to reconcile God's justice, wisdom and power with the apparent presence of evil and imperfection in the world. His denial of a providential God would, by itself, seem to rule out any Spinozistic approach to the issue. However, Spinoza does have a good deal to say about the theodicy question. By denying the personal immortality of the soul, Spinoza, I believe, self-consciously offers a response to a traditional approach found within medieval Jewish philosophy, one which relies on the rewards for virtue to be expected in the "world to come". Moreover, the constituents of Spinoza's approach are, in fact, found in that earlier tradition, and Spinoza's views are a natural extension of a trend within medieval Jewish rationalism.
\end{abstract}

L'idée même de discuter de l'approche spinoziste du problème de la théodicée doit paraître $a$ priori hors de propos, si ce n'est carrément absurde. Après tout, Spinoza ne rejette-t-il pas toute la question de la théodicée sur la base de son incohérence et de son enracinement dans une conception fausse ou inadéquate de la nature des choses ? Il semblerait, de fait, que la question ne puisse même pas être posée à l'intérieur de son système métaphysique et moral, et qu'il soit par conséquent inutile de chercher à comprendre autre chose que les raisons de cette impossibilité. Et pourtant, comme j'espère le montrer, je crois que ce n'est pas tout ce qu'on peut en dire.

1.

Pour que prenne naissance le projet même d'une théodicée, un certain nombre de conditions doivent être réunies. Premièrement, il faut qu'on croie qu'il y a un Dieu et que Dieu est le créateur (ou, du moins, la source causale) du monde 
dans lequel on vit. Deuxièmement, il faut qu'on croie qu'il y a du mal (qu'il soit apparent ou réel) dans la création divine. Qu'on veuille l'appeler «mal moral ", " mal métaphysique » ou encore " mal physique ", pour reprendre la catégorisation de Leibniz, il faut tout du moins qu'il y ait un certain type d'imperfection dans le monde, en particulier vis-à-vis des êtres humains. Parfois, ces imperfections seront les péchés commis par les agents moraux, tandis qu'à d'autres moments l'imperfection consistera dans la souffrance de l'innocent et le triomphe des méchants. Les défauts de naissance, les désastres naturels et les châtiments non mérités sont autant de caractéristiques indéniables et, apparemment du moins, inexplicables du monde. Or, en elles-mêmes et pour elles-mêmes, ces choses ne sont pas problématiques. Elles ne le deviennent - et génèrent alors l'ensemble des questions connues, à la suite de Leibniz, sous le nom de " théodicée " - que lorsqu'on les met en relation avec certaines affirmations sur Dieu qui nous empêchent de répondre à ces questions de manière trop facile ou simpliste. La première de ces affirmations stipule que Dieu est tout-puissant, c'est-à-dire que Dieu peut faire tout ce qu'il veut et que la volonté de Dieu est, du moins absolument parlant, d'une portée infinie. Cette théorie nous empêche de dire que Dieu ne peut rien contre les maux de sa création. La seconde de ces affirmations dit que Dieu est omniscient, c'est-à-dire qu'il connaît tout, y compris les défauts soi-disant apparents de son œuvre. Cette conception nous empêche de dire que Dieu pourrait (et voudrait) faire quelque chose contre le mal présent dans sa création si seulement il en avait connaissance, mais qu'étant donné que ce n'est évidemment pas de lui que ce mal provient, il n'en connaît tout simplement pas l'existence. La troisième affirmation pose que Dieu est bienveillant et juste, ou encore que Dieu ne veut que ce qui est bon. On ne peut alors résoudre l'énigme en disant tout simplement que Dieu connaît le mal et qu'il serait capable de l'empêcher, mais qu'il ne se soucie pas de le faire. Comment, dès lors, peut-on concilier l'existence du mal, de la douleur et de la souffrance dans le monde, avec le fait que le monde a été créé par un Dieu à la fois juste, sage, bon, omniscient, toutpuissant et libre?

Spinoza rejette un certain nombre de ces affirmations. Tout d'abord, pour Spinoza, Dieu ou la Nature (Deus sive Natura, selon la fameuse phrase que ses amis ont tirée de l'édition hollandaise posthume de ses œuvres) n'est pas tout-puissant dans le sens classique du terme. Si le Dieu de Spinoza est bien la cause infinie et première de tout ce qui existe, il n'est pas, en revanche, un Dieu libre agissant par volonté et choix. Comme il le dit explicitement, Dieu, tout en étant libre, «ne produit rien par effet d'une liberté de la volonté » ${ }^{1}$ :

1. Spinoza dit bien que Dieu est « libre », mais seulement parce que « Dieu agit par les seules lois de sa nature et sans subir aucune contrainte " (Éthique IP17), et non parce que Dieu serait doté d'une « liberté de la volonté ». Toutes nos références à l'Éthique suivent la notation standard en partie (chiffres romains), proposition (P) et, le cas échéant, scolie (S). La traduction utilisée est celle de Charles Appuhn, Spinoza, CEuvres, 4 volumes, GF-Flammarion, 1965. 
tous les aspects de l'univers suivent nécessairement et avec une détermination absolue de la substance infinie, à savoir, Dieu et ses attributs. De plus, le Dieu de Spinoza n'est pas non plus un être bon et juste. En fait, Dieu est, pour Spinoza, totalement dépourvu de toute caractéristique morale. Dieu est la nature, ou du moins les aspects actifs, génératifs, éternels et infinis de la nature - ce qu'il appelle la Natura naturans -, et ce n'est pas un être motivé à agir par une quelconque conception du bien. De fait, le Dieu de Spinoza n'agit en vue d'aucune fin de quelque sorte que ce soit. Il n'y a pas de téléologie, ni à l'intérieur de la nature, ni pour la nature dans son ensemble. Assigner à Dieu une " bonne fin en vue de laquelle il agit » ou une volonté libre mue par une conception du bien équivaudrait pour Spinoza à succomber au type d'anthropomorphisation de Dieu qui est typique des superstitions organisées qui se font passer pour les plus grandes religions.

Sans un Dieu libre, bon et juste, toute la question de la théodicée semble ne même pas pouvoir être posée - ou il serait peut-être plus juste de dire que l'on répond immédiatement à la question. S'il y a de la souffrance et des désastres dans le monde, c'est parce qu'il n'existe pas de Dieu sage et providentiel qui veillerait sur le monde, et qu'au contraire, dans ce monde, tous les événements sont déterminés simplement par les lois de la nature. Dans ce cas, alors, pourquoi faudrait-il même s'intéresser à Spinoza dans le contexte du problème de la théodicée ?

De plus, Spinoza lui-même semble parfois n'avoir que du mépris pour ceux qui perdent leur temps à essayer de résoudre le problème du mal en élaborant une théodicée (un peu à la manière des amis de Job qui essayaient de lui expliquer les raisons de sa souffrance).

Considérez, je vous le demande, où les choses en sont enfin venues! Parmi tant de choses utiles offertes par la Nature, ils n'ont pu manquer de trouver bon nombre de choses nuisibles, telles les tempêtes, les tremblements de terre, les maladies, etc., et ils ont admis que de telles rencontres avaient pour origine la colère de Dieu excitée par les offenses des hommes envers lui ou par les péchés commis dans son culte; et, en dépit des protestations de l'expérience quotidienne, montrant par des exemples sans nombre que les rencontres utiles et les nuisibles échoient sans distinction aux pieux et aux impies, ils n'ont pas pour cela renoncé à ce préjugé invétéré. Ils ont trouvé plus expédient de mettre ce fait au nombre des choses inconnues dont ils ignoraient l'usage, et de demeurer dans leur état actuel et natif d'ignorance, que de renverser tout cet échafaudage et d'en inventer un autre. Ils ont donc admis comme certain que les jugements de Dieu passent de loin la compréhension des hommes (Éthique I, Appendice).

S'engager dans la spéculation de la théodicée n'est faire preuve que de superstition, et non d'entendement.

Ce que je voudrais montrer, c'est qu'en fait, Spinoza n'était pas entièrement insensible au problème du mal et de la souffrance. Mais ce qu'il nous offre, en revanche, n'est pas tant une théodicée qu'une réponse à un type particulier de tentative de théodicée. Ceci, en soi, n'est pas particulièrement nou- 
veau ou surprenant, et n'importe quel lecteur attentif de l'Éthique peut s'en rendre compte par soi-même. Mais ce que je trouve vraiment tout particulièrement intéressant, ce sont les deux choses suivantes : premièrement, que le type de théodicée auquel Spinoza s'attaque soit d'un genre très répandu dans la philosophie juive médiévale et même dans des textes rabbiniques plus anciens ; et deuxièmement, que les germes de la réponse spinoziste à ce type de théodicée soient elles aussi présentes dans la pensée médiévale juive du mal. Je voudrais proposer ici l'idée qu'en élaborant sa conception du bonheur humain, Spinoza ne fait rien d'autre qu'offrir une reductio d'une théodicée particulière que l'on retrouve dans le rationalisme juif. Au fond, ce que je crois que Spinoza veut dire, c'est que certains penseurs juifs classiques ont bien vu les choses, mais qu'ils n'ont pas poussé leur conception assez loin ( $\mathrm{du}$ moins, pas explicitement).

\section{2.}

Avant d'en venir à ma thèse principale, qu'on me permette d'envisager deux éléments dans la pensée de Spinoza qui ne sont pas à proprement parler centraux pour ma discussion, mais qui portent néanmoins sur le problème de la théodicée.

Tout d'abord, il y a la définition que donne Spinoza des termes de « bien » et de « mal ». On sait que Spinoza affirme que le bien et le mal ne sont " rien de réel en eux-mêmes ». Absolument parlant, il n'y a pas de " défaut » de la nature :

La perfection donc et l'imperfection ne sont, en réalité, que des modes de penser, je veux dire des notions que nous avons accoutumé de forger parce que nous comparons entre eux les individus de même espèce ou de même genre [...]. Nous les appelons imparfaits, parce qu'ils n'affectent pas notre âme pareillement à ceux que nous appelons parfaits, et non parce qu'il leur manque quelque chose qui leur appartienne ou que la Nature ait péché (Éthique IV, Préface).

Les qualifications de «bon " et de " mauvais " sont seulement relatives à notre conception des choses, et ne dénotent rien de réel à propos des choses elles-mêmes. "Quant au bon et au mauvais, ils n'indiquent également rien de positif dans les choses, considérées du moins en elles-mêmes, et ne sont autre chose que des modes de penser ou des notions que nous formons parce que nous comparons les choses entre elles ». Par "bon », tout ce qu'on entend, c'est « ce que nous savons avec certitude qui est un moyen de nous rapprocher de plus en plus du modèle de la nature humaine que nous proposons ». À l'inverse, par " mauvais ", tout ce qu'on entend est seulement " ce que nous savons avec certitude qui nous empêche de reproduire ce modèle " (Éthique IV, Préface). En d'autres termes, ce qui est bon, c'est simplement ce qui est considéré par une créature comme utile ; ce qui est mauvais, c'est simplement 
ce que celle-ci considère comme un obstacle à la réalisation de ses fins ou à l'accomplissement de ses désirs. Rien n'est bon ou mauvais sauf dans la mesure où on le juge bon ou mauvais.

Le problème, toutefois, c'est que Spinoza parle plus loin de «la connaissance vraie du bien et du mal ", ce qui suggère bien entendu que l'on peut avoir raison ou se tromper sur ce qui est vraiment bon ou utile ${ }^{2}$. Et effectivement, la philosophie morale de Spinoza exige qu'il y ait une certaine sorte de recherche de ce qui est réellement notre bien en tant qu'êtres rationnels à savoir, la recherche de l'acquisition d'idées adéquates et du troisième genre de connaissance. Le bon et le mauvais peuvent être relatifs à un modèle ou une norme quelconques placés devant nos yeux - dans ce cas, un modèle de l'être humain -, mais il existe aussi un modèle spécifique et objectif de l'être humain, à savoir "l'homme libre " ou "le vertueux ", que nous devons nous efforcer d'imiter. « La connaissance de Dieu », écrit Spinoza en Éthique IVP28, est «le bien suprême de l'âme ». Et elle n'est pas seulement bonne pour la raison qu'on croit qu'elle est favorable à notre bien-être et qu'elle est un soutien pour notre conatus, mais bien parce qu'il en est réellement ainsi. Assurément, cette « connaissance vraie du bien et du mal » est tout autant éprouvée affectivement que la conception purement subjective du bien et du mal, ou du moins, elle "enveloppe " une composante affective qui fait son travail de persuasion ; mais je ne crois pas qu'elle puisse être facilement écartée sous le motif d'être " simplement relative " à nos conceptions. Il s'agit là d'un problème connu de l'interprétation de la théorie éthique de Spinoza, et je ne veux pas y entrer trop profondément $\mathrm{ici}^{3}$. Mais ce qui, d'après moi, est bel et bien montré par cela, c'est que la résolution par Spinoza - ou l'écartement - du problème de la théodicée ne consiste pas en une élimination pure et simple de la réalité du bien et du mal ou en une réduction de ceux-ci à de simples modes de notre pensée.

L'autre point que je voudrais mentionner ici sans m'y attarder, concerne une autre dimension du problème de la théodicée. Parfois ce problème n'est pas formulé en termes de " pourquoi, dans un monde créé par un Dieu bon, puissant et omniscient, le péché et la souffrance existent et pourquoi toutes les créatures ne peuvent parvenir à jouir de la plus haute perfection qu'ils méritent ", mais plutôt et avant tout, en termes de " pourquoi Dieu a créé un monde distinct de lui ». Si Dieu est parfait et auto-suffisant, qu'estce qui a bien pu le conduire à créer quelque chose en dehors de lui ? Or pour Spinoza, cette question n'a même pas à être posée. Premièrement, Dieu ne choisit pas du tout de créer ou pas; le monde découle nécessairement des

2. Voir, par exemple, IVP14.

3. Voir la discussion de ce point par Curley, Edwin, Behind the Geometrical Method: A Reading of Spinoza's Ethics, Princeton, Princeton University Press, 1988, p. 120-123, et par Allison, Henry E., Benedict de Spinoza : An Introduction, New Haven, Yale University Press, 1987, p. 140-144. 
attributs éternels et des modes infinis, et n'aurait pas pu ne pas exister. Il n'y a jamais eu de temps avant lequel il n'existait pas et à partir duquel il est advenu à l'être. Deuxièmement, pour Spinoza, le monde n'est pas, en fait, séparé de Dieu. Au contraire, Dieu est précisément la substance de l'univers, la cause immanente - et non une cause transitive distincte - de tout ce qui existe.

Il y a, bien entendu, une longue tradition consacrée qui a pensé de cette manière la relation entre Dieu et la création, tant dans la philosophie juive que dans la philosophie arabe, et même, auparavant, dans la pensée grecque. Et il serait très intéressant de reprendre le projet de Wolfson de chercher dans ces traditions les sources du rejet spinoziste de cet aspect du problème de la théodicée ${ }^{4}$. Mais dans le cadre de cet essai, je dois laisser de côté ce projet.

\section{3.}

Lorsque Job est submergé par la douleur, lorsqu'on lui a volé tout ce qui lui était cher, lorsque, finalement, tout semble perdu, il élève la voix pour se plaindre à Dieu du traitement qu'il a reçu, lui qui est selon toutes les apparences un homme de bien. Ses amis viennent essayer de lui offrir leur consolation ou, du moins, une rationalisation visant à expliquer pourquoi il a été l'objet d'un tel désastre. D'après eux, il doit y avoir une raison à l'affliction de Job, résidant ou bien dans un péché commis par lui ou l'un des membres de sa famille, ou bien dans une raison que Dieu posséderait mais qui excéderait les pouvoirs de notre connaissance. L'un de ses compagnons croit que l'on ne devrait pas limiter notre jugement de la justice divine à ce qu'on en voit dans cette vie, où souvent le juste est opprimé et le méchant prospère. Bildad suggère que si Job est réellement innocent, il doit prendre en considération le fait qu'il sera en bout de ligne récompensé - et pas seulement dans cette vie, qui pour le juste est longue, mais tout particulièrement dans ce qui lui arrivera après la mort :

C'est la lumière du méchant qui s'éteindra! La flamme, à son foyer, cessera de briller. La lumière, chez lui, perdra de sa clarté, la lampe de sa vie va s'éteindre à son tour [...]. Alors il deviendra comme un arbre qui meurt, de la racine, en bas, jusqu'aux rameaux, en haut. Au pays, on perdra tout souvenir de lui, son nom ne sera plus prononcé nulle part. De la lumière, on l'expulsera dans la nuit, et on le chassera de la terre habitée. Aucun enfant n'assurera sa descendance, aucun ne survivra, de toute sa maison (Job, 18, 5-19).

La destinée ultime du méchant, malgré sa prospérité temporaire, est l'oubli. La conséquence en est que le juste, quant à lui, tout en souffrant peutêtre durant sa vie, devrait se réjouir à l'idée que les fruits de sa vertu persis-

4. Wolfson, Harry, The Philosophy of Spinoza, Cambridge, Harvard University Press, 1934, 2 volumes. 
teront bien après qu'il ait quitté ce monde. Comme Sofar l'affirme avec insistance, " les méchants ne crient victoire que peu de temps, la joie est de courte durée chez l'homme de mauvaise foi. Même s'il est de taille à monter jusqu'au ciel, même s'il a la tête au niveau des nuages, il finira comme ses propres excréments » (Job, 20, 5-7).

Ce texte biblique ne comporte aucune mention explicite et même aucune implication d'une âme immortelle ou d'une vie après la mort, éléments qui n'apparaissent que plus tard dans le judaïsme. Mais il suggère bel et bien une théodicée qui a la structure générale suivante : ne jugez pas la justice divine avant d'avoir adopté une perspective à long terme sur la destinée d'une personne, y compris sur sa mort et sur ce qui se passe après.

Lorsque le judaïsme finit par développer, au cours de la période rabbinique, une conception bien définie de l'immortalité de l'âme et de la résurrection des morts, qui accompagne les idées concomitantes de récompense éternelle au paradis (ou Gan Eden) et de punition éternelle en enfer (Sheol ou Gehinnom), ce message fondamental peut enfin prendre la forme d'une théodicée pleine et entière. Dès lors, on considère que le véritable domaine de la justice divine n'est pas $c e$ monde, mais le monde à venir, Olam ha-Ba. C'est là, et à ce moment-là, que les vraies récompenses et les vrais châtiments sont assignés aux justes et aux mauvais. Les innocents peuvent bien souffrir dans cette vie-ci, ils peuvent bien être affligés par la peine et le malheur, et peut-être même à cause de leurs quelques péchés, mais ils seront plus que dédommagés pour leurs souffrances dans l'autre vie. Inversement, les méchants peuvent bien prospérer en ce monde, et le plus souvent aux dépens des justes, mais tout ce qu'ils peuvent gagner ici et maintenant n'est rien en comparaison des souffrances qui leur seront infligées après leur mort ou à la fin des temps. Lorsqu'on prend tout cela en considération, on peut comprendre dans quel contexte plus large vient s'inscrire la souffrance des innocents, et, ce qui importe plus, on peut comprendre la véritable justice des voies divines. Lorsqu'on adopte une perspective à long terme, on constate que chacun reçoit finalement bien ce qu'il mérite, et l'on n'est plus tenté de questionner la bonté, la sagesse et le pouvoir de Dieu. On trouve même des auteurs dans la littérature du Talmud et des Midrashim pour qui la souffrance dans cette vie apparaît comme un prélude à la sainteté dans le monde à venir ${ }^{5}$. Quoique ce ne soit pas là le courant dominant de la théodicée dans la pensée juive - de nombreux rabbins, croyant que la justice divine se manifeste non pas dans le monde à venir, mais dans le monde dans lequel on vit, ont insisté sur l'importance du châtiment et de la récompense pour le péché et la vertu dans cette vieci -, il faut reconnaître que cette conception a exercé un pouvoir d'attraction

5. Voir, par exemple, les paroles de R. Nehemia : "Quel est le chemin qui conduit un homme vers la vie dans le monde à venir ? Les souffrances ", Sifre Deuteronomy, sect. 32 ; f.73b. 
puissant sur d'importantes autorités religieuses et sur des philosophes juifs majeurs.

Une théodicée de ce type est formulée en des termes particulièrement clairs et systématiques par le philosophe du dixième siècle Saadia ben Joseph (ou Saadia Gaon), qui était également le directeur (ou gaon) de l'académie à Babylone. Si Dieu est juste, demande Saadia, comment se fait-il que l'on voie des personnes pieuses être affligées par la douleur et le malheur dans cette vie tandis que, dans le même temps, l'impie prospère ? Saadia considère son explication comme la seule explication rationnelle, c'est-à-dire comme la seule qu'on puisse raisonnablement attribuer à un Dieu rationnel et non arbitraire. Une personne " pieuse », dit-il, est quelqu'un dans la conduite de qui " les bonnes actions dominent ", tandis qu'un « impie » est quelqu'un dans la conduite de qui la plupart des actions sont mauvaises. Il arrive que des personnes pieuses commettent un plus grand nombre de péchés que d'autres (ces péchés ne constituant toujours qu'une minorité de leurs actions dans l'ensemble), et qu'à l'inverse certains impies commettent plus de bonnes actions que d'autres personnes. Cependant il existe, affirme-t-il avec insistance, un " deuxième monde » au-delà de celui-ci, "le monde des rétributions ». Il n'advient « qu'une fois que le nombre total d'êtres rationnels est atteint. C'est alors que [Dieu] rétribue tous [les êtres rationnels] selon leurs actions " ${ }^{6}$. Mais il serait injuste de ne pas prendre en compte toutes les actions d'une personne, autant celles qui sont en minorité que celles en majorité. Saadia prétend donc que Dieu a établi une loi générale selon laquelle tous les individus seront payés en retour dans ce monde-ci pour la plus petite partie de leurs actions, et dans le monde à venir pour la majorité de leurs actions. " Il n'a par conséquent institué de récompense dans ce monde-ci que pour la plus petite partie de la conduite d'une personne [...], tandis que la totalité de ses mérites est réservée pour un temps très éloigné ${ }^{7}$. Ceci explique

pourquoi il arrive souvent qu'une personne globalement vertueuse soit sujette à beaucoup de défaillances, à cause desquelles elle mérite d'être au supplice pendant la plus grande partie de sa vie. D'un autre côté, une personne globalement impie peut avoir à son actif beaucoup de bonnes actions, pour lesquelles elle mérite de jouir du bien-être pendant la plus grande partie de son existence terrestre.

Une fois que tout est dit et fait, chacun reçoit exactement ce qu'il mérite : chacun reçoit des récompenses et des punitions, soit dans ce monde-ci soit dans le suivant, parfaitement proportionnées à ses actions. Enfin, Saadia admet qu'il arrive parfois qu'une personne pure de tout péché souffre néanmoins dans ce monde-ci, mais il répond tout simplement en disant qu'elle sera récompensée de ses peines dans le monde à venir.

6. Saadia ben Joseph, Le livre des croyances et des opinions, traité $\mathrm{V}$, chapitre 1.

7. Ibid., chapitre 2 . 
Des philosophes juifs médiévaux plus tardifs tels que Maïmonide et Gersonide s'accordent avec Saadia pour dire que la vraie récompense de la vertu n'est pas l'absence de douleur ou de souffrance dans cette vie. Et ce, même si une vie de vertu véritable doit, de par la nature de la vertu, préserver et soulager de beaucoup de vicissitudes. Les bons trouvent leur véritable récompense dans le monde à venir, dans la vie d'après. C'est là également, bien sûr, une caractéristique récurrente dans plusieurs théodicées chrétiennes ; mais ce qui m'intéresse tout particulièrement pour l'étude de Spinoza, c'est le tournant intellectualiste que les rationalistes juifs Maïmonide et Gersonide ont donné à cette doctrine. Quoique je croie que la plupart des choses que j'ai à dire soient vraies aussi de Maïmonide, j'entends me concentrer ici seulement sur Gersonide (Levi ben Gershom), le rabbin et philosophe du XIV siècle vivant en Provence.

Dans son œuvre philosophique principale, Les Guerres du Seigneur (en hébreu, Sefer Milchamot ha-Shem), Gersonide soutient que l'on ne doit pas juger la providence divine en se basant seulement sur ce qu'on observe dans ce monde, et en particulier sur la distribution du bien et du mal ${ }^{8}$. Car ce qu'on voit généralement, c'est clairement un manque d'ordre et d'équité dans la distribution de ceux-ci, avec des personnes qui ne reçoivent apparemment pas leur dû. Le mal arrive aux justes, et le bien aux pécheurs. Par conséquent, écrit-il, "ou bien Dieu pouvait établir un déterminisme tel que l'homme soit rétribué selon ses œuvres, mais il n'a pas fait cet effort (il serait alors indubitablement injuste, qu'à Dieu ne plaise) ; ou bien Dieu n'en était pas capable (c'est encore une imperfection en lui !) ${ }^{9}$.

La solution à cette énigme est que "la récompense et le châtiment véritables ne consistent pas en ces biens et en ces maux perceptibles : en effet, la récompense et le châtiment pour l'homme en tant qu'homme, doivent être des biens et des maux spécifiquement humains, mais non pas des biens et des maux qui ne le sont pas ${ }^{10}$. Les biens et les maux principaux en ce monde sont les bénéfices et les pertes de type matériel, tels que de bons aliments et d'autres objets et plaisirs sensuels, lesquels nous sont communs avec d'autres créatures. Or ceux-ci peuvent très bien ne pas être distribués en fonction du mérite de la personne, mais par le hasard et la nature. Le "véritable " bien humain, en revanche, consiste dans " l'acquisition du bonheur spirituel ", qui représente la perfection la plus haute et la plus intrinsèque à un être humain. «Le malheur pour l'homme c'est d'être privé de la béatitude de l'âme, qui perd ainsi son entéléchie, il est donc évident que, pour l'homme en tant

8. Ce n'est pas là le seul endroit où Gersonide traite de la question de la providence et du problème du mal. Le sujet est également abordé, d'une manière très similaire, dans son Commentaire sur le Livre de Job. La meilleure discussion de Gersonide sur la providence se trouve dans l'étude magnifique de Touati, Charles, La pensée philosophique et théologique de Gersonide, Paris, Éditions de Minuit, 1973, parties 6 et 7.

9. Guerres du Seigneur, livre IV, chapitre 6, traduction par Charles Touati, Les Guerres du Seigneur, Livres III et IV, Paris, Mouton \& Co, 1968, p. 145.

10. Ibid., p. 146. 
qu'homme, la récompense ou le châtiment, ce sont la béatitude ou l'absence de béatitude de l'âme, mais non pas ces biens et ces maux perceptibles qui procèdent des Corps Célestes ${ }^{11}$.

Ce en quoi consiste ce bonheur spirituel, d'après Gersonide, c'est dans l'acquisition de la connaissance et dans l'union avec un intellect supérieur. Laissé à lui-même, l'esprit humain est limité à la connaissance du monde autour de lui tel qu'il peut être perçu par son intellect matériel, qui travaille en conjonction avec les sens. Mais par l'entremise de l'Intellect Agent, c'està-dire de l'Intellect (ou Âme) séparé qui gouverne ce monde et la sphère la plus profonde à laquelle il appartient, l'esprit humain peut parvenir à la connaissance de vérités plus générales. Il peut connaître des vérités éternelles qu'il tire de l'expérience à l'aide de l'Intellect Agent. C'est ainsi que l'esprit humain passe de la connaissance sensible par images à l'appréhension des intelligibles, des formes des choses en dehors de leur matérialité12. Par ce processus, l'esprit humain parvient à comprendre l'ordre véritable du monde, et sa connaissance grandit jusqu'à être le reflet de la connaissance qui est dans l'Intellect Agent lui-même.

Or ces vérités éternelles que l'esprit peut saisir constituent son " intellect acquis », une partie de l'esprit qui est différente de l'intellect matériel. L'âme humaine, pour Gersonide, est simplement une partie du corps, elle n'est pas une substance séparée ou incorporelle. De la sorte, l'âme humaine elle-même est corruptible ${ }^{13}$. Mais l'intellect acquis, lui, du fait qu'il n'est composé que d'une connaissance éternelle, n'est pas corruptible. En réalité, il est séparable du corps et de l'intellect matériel. Parce qu'il n'est rien d'autre que " la connaissance de l'ordre même inhérent à l'Intellect Agent » — un ordre de la nature qui s'exprime dans le monde, lequel est gouverné par cet Intellect -, l'intellect acquis est éternel et immatériel. Lorsque le corps périt, l'âme humaine et l'acquisition de connaissances font de même, mais l'intellect acquis demeure. Il est, comme le rappelle Gersonide, immortel. En fait, l'immortalité d'un être humain ne consiste qu'en la persistance de son intellect acquis après la mort du corps ${ }^{14}$.

Or, le bonheur humain véritable réside dans l'accomplissement intellectuel représenté par le perfectionnement de l'esprit, soit la connaissance obtenue par l'intellect acquis. Et dans le cours de cette vie, nous pouvons jouir d'une partie de cette perfection, mais les exigences du corps et la force des circonstances empiriques viennent souvent se mettre en travers du chemin de l'accomplissement et de la jouissance de la vraie perfection. Ainsi, même les

11. Ibid., p. 146. Cela ne veut pas dire pour autant que Gersonide était indifférent à la distribution de ces biens « imaginaires » dans ce monde-ci. En réalité, une partie de son propos sur la providence inclut un passage consacré entièrement à cette question, qui repose aussi sur celle de l'union avec l'intellect actif.

12. Voir Gersonide, Guerres, Livre I, chapitre 10.

13. Voir Gersonide, Guerres, Livre I, chapitre 11.

14. Ibid. 
vertueux, ceux qui ont consacré leur vie à la recherche de la connaissance véritable, sont soumis à la fortune, aux dérangements et aux imperfections de ce monde - mais à leur mort, par contre, ils sont en mesure de jouir de leur plus grand bonheur, et ce au plus haut degré.

Il importe de réaliser que tout homme qui a atteint cette perfection jouit du bonheur résultant de sa connaissance après la mort. Nous pouvons avoir une première idée de ce plaisir grâce à celui que nous procure le peu de connaissance que nous avons maintenant et qui domine la partie animale de notre âme [de telle sorte que] l'intellect est isolé dans son activité. Ce plaisir est incomparable aux autres plaisirs et n'a aucune relation d'aucune sorte avec eux. C'est pourquoi ce plaisir sera d'autant plus grand après notre mort, car alors toute la connaissance que nous avons acquise dans cette vie sera contemplée de manière continue et l'on appréhendera simultanément toutes les choses présentes dans notre esprit, puisqu'après la mort, ce qui faisait obstacle à ce genre de connaissance, c'est-à-dire la matière, aura disparu [...]. Après la mort, [l'intellect] détiendra de manière simultanée toute la connaissance qu'il aura acquise durant la vie ${ }^{15}$.

La vraie récompense de la vertu, de la recherche de la connaissance et de l'excellence intellectuelle, sera donnée dans le monde à venir, et non dans une vie dénuée de mal et de souffrance dans ce monde : notre bonheur le plus haut n'est donné qu'après la mort. « D’après nos Maîtres, la récompense et le châtiment véritables sont réservés au monde futur et [...] il n'est aucunement nécessaire que la récompense et le châtiment, consistant en biens et en maux corporels, soient proportionnés ici-bas à la vertu et à la méchanceté. Ils ont déclaré : "Dans ce monde, point de salaire pour une bonne action !" ${ }^{16}$.

\section{4 .}

Étant donné la conception spinoziste de la connaissance et du bonheur humain, on peut dire que Spinoza dispose de tous les éléments pour élaborer une théodicée du même type que celle présentée par Gersonide. En fait, il est même assez clair que sa conception de la connaissance et du bonheur a été fortement influencée par ce qu'il avait lu chez Gersonide et Maïmonide ${ }^{17}$. Mais

15. Gersonide, Guerres, Livre I, chapitre 13.

16. Gersonide, Guerres, Livre IV, chapitre 6, p. 163. Comme nous l'avons dit plus haut, ce n'est pas là le seul type de théodicée que l'on trouve dans la pensée juive. De nombreux rabbins, croyant que la justice divine se manifeste non dans le monde à venir mais dans ce monde-ci, ont insisté sur l'importance des punitions et des récompenses pour le péché et la vertu dans cette vie. Il a même été soutenu que, en fait, la vision de Gersonide que nous venons de voir allait à l'encontre de la tradition rabbinique (cf. Kellner, Menachem, "Gersonides, Providence and the Rabbinic Tradition ", Journal of the American Academy of Religion, 42, 1974).

17. Parmi les livres retrouvés dans la bibliothèque de Spinoza après sa mort se trouvait une copie du Moreh Nevukhimde Maïmonide, c'est-à-dire la traduction en hébreu du Guide des Égarés, mais aucun exemplaire d'un livre de Gersonide (cf. Catalogus van de Bibliotheek 
il est tout aussi clair, selon moi, que Spinoza niait l'immortalité de l'âme et que, de ce fait, il s'empêchait de s'engager dans un type particulier de théodicée - à savoir, dans celle promue par les rabbins du Talmud, par Saadia, Gersonide et tout un ensemble d'autres philosophes, juifs ou gentils. S'il est vrai qu'il n'y a aucune vie après la mort, s'il est vrai que l'on ne persiste pas après la corruption du corps et la fin de l'existence dans la durée, alors il ne peut exister aucune récompense à espérer (ni aucune punition à craindre) dans aucun « monde à venir » que ce soit. L'idée que je voudrais proposer ici, c'est que Spinoza, en réalité, s'engage dans une sorte de reductio de la théodicée de Gersonide : étant donné tout ce que dit Gersonide à propos de l'âme, de son accomplissement intellectuel et de son bonheur - et avec quoi Spinoza est entièrement d'accord -, il ne peut y avoir aucune récompense véritable de la vertu dans un monde qui serait, prétendument, après celui-ci. Bien plutôt, la vertu doit être à elle-même sa propre récompense dans ce monde, en tant que source d'un bonheur permanent et d'un dégagement des vicissitudes du hasard et de la fortune. En d'autres termes, Spinoza mène la théodicée de Gersonide jusqu'au bout de ce qui lui paraît en être la conclusion logique.

Le projet principal de l'Éthique consiste à montrer comment l'on peut parvenir, dans un monde de déterminisme, à l'autonomie et au bonheur dans une certaine mesure. Plus particulièrement, Spinoza veut expliquer comment nous sommes généralement les esclaves de nos passions, ballottés sur les flots de nos réactions affectives face au monde. C'est dans l'exacte mesure où l'on accepte de rester dans cet état que l'on est sujet au contrôle de forces en dehors de notre contrôle, aux objets éternels qui vont et qui viennent selon la manière dont les lois de la nature les y déterminent. Et tant que l'on identifie notre bonheur à la possession des objets et états de choses temporels et changeants auxquels on accorde de la valeur, le bonheur reste une chose incontrôlable et instable.

Cependant, si l'on on ne peut pas contrôler la nature, on peut contrôler notre réponse à la nature. On peut tenter de réduire le pouvoir que nos passions ont sur nous et, ce faisant, parvenir à un minimum de liberté, à savoir, d'indépendance eudémoniste aux forces extérieures qui agissent sur nous ${ }^{18}$. Le moyen pour cela, c'est la connaissance. C'est en nous comprenant nousmêmes et le monde, et en particulier en voyant comment toutes les choses sont

\footnotetext{
der Vereniging Het Spinozahuis te Rijnsburg, Leiden, Brill, 1965). Pourtant, on ne peut aucunement douter du fait que Spinoza avait lu et connaissait bien au moins le Sefer Milhamot haShem, si ce n'est les commentaires bibliques de Gersonide. Pour un approfondissement des relations entre Spinoza et Maïmonide, Voir Roth, Léon, Spinoza, Descartes and Maimonides, Oxford, Oxford Clarendon Press, 1924, Harvey, Warren Zev, "A Portrait of Spinoza as Maimonidean ", Journal of the History of Philosophy 19, 1981, et les analyses de Harry Wolfson dans The Philosophy of Spinoza (op. cit.).

18. Il ne s'agit alors pas, cependant, d'une indépendance physique réelle ou même psychologique pleine, car comme Spinoza le rappelle bien, aucun être humain ne peut être complètement en dehors de la chaîne causale de la nature (Voir Éthique IVP4).
} 
reliées aux natures éternelles qui constituent la substance unique - Dieu ou la Nature - et comment elles suivent nécessairement de ces natures ou attributs, que l'on peut progresser vers une paix de l'âme de type stoïcien : la force des passions diminuera et nous serons moins atteints par les vicissitudes du monde autour de nous ${ }^{19}$. Autrement dit, nous nous rapprocherons d'un état permanent de joie et de contentement. Ce vers quoi nous devons tendre, dans les termes de Spinoza, c'est vers " la connaissance du troisième genre ", une compréhension intuitive des choses du point de vue de l'éternité, par opposition à la connaissance partielle et lacunaire des choses que nous avons par nos sens et notre imagination.

La connaissance du troisième genre appréhende les choses non dans leurs relations causales finies, particulières et changeantes, ou dans leur existence dans la durée, mais à travers leur essence, leur nature immuable. Et comprendre les choses de manière essentielle de cette façon revient à les mettre en relation avec leurs causes infinies, savoir la substance (Dieu) et ses attributs :

Les choses sont conçues par nous comme actuelles en deux manières : ou bien en tant que nous en concevons l'existence avec une relation à un temps et à un lieu déterminés, ou bien en tant que nous les concevons comme contenues en Dieu et comme suivant de la nécessité de la nature divine. Celles qui sont conçues comme vraies ou réelles de cette seconde manière, nous les concevons avec une sorte d'éternité, et leurs idées enveloppent l'essence éternelle et infinie de Dieu (Éthique VP39S).

Ce à quoi nous nous attachons, c'est à comprendre les corps à travers l'étendue et ses lois et non à travers d'autres corps. Pour utiliser l'expression de Spinoza, nous nous efforçons de comprendre les choses "sub specie aeternitatis ", c'est-à-dire de les voir non dans une perspective finie, mais dans la perspective infinie et éternelle qui est celle du point de vue divin.

Je crois que la connaissance du troisième genre dont parle Spinoza est la même chose que l'intellect acquis dont parle Gersonide. Comme Gersonide, Spinoza identifie cette connaissance avec la recherche d'une perspective générale, asensorielle et atemporelle, et avec une compréhension en profondeur de l'ordre de la nature. Il s'agit d'une appréhension intellectuelle qui réside dans la possession d'idées claires et distinctes (ou adéquates) des choses, et Spinoza précise que la recherche de cette connaissance est également ce qui constitue la « vertu » et la vie bonne ${ }^{20}$. Mieux encore, comme l'intellect acquis chez Gersonide, la connaissance du troisième genre est éternelle, et ce fondamentalement parce qu'elle est la connaissance de Dieu lui-même. Lorsque le corps et ses facultés meurent, cette connaissance, elle, qui ne forme pour l'instant qu'une partie de notre bagage de connaissances, demeure.

19. C'est l'aboutissement des parties IV et V de l'Éthique. Voir en particulier VP6 : "Dans la mesure où l'Âme connaît toutes choses comme nécessaires, elle a sur ses affects une puissance plus grande, c'est-à-dire qu'elle en pâtit moins ".

20. Voir Éthique IVP23-26. 


\section{Philosophiques / Printemps 2002}

Mais c'est précisément à ce point que Spinoza commence son offensive. À partir de ces mêmes doctrines, Spinoza va dire que l'immortalité personnelle de l'âme n'existe pas - et, étant donné la similitude de sa conception de la connaissance et du bonheur avec celle de Gersonide, on peut bel et bien penser qu'il veut dire que Gersonide aurait dû la nier également. S’il est vrai que tout ce qui survit après la mort du corps est un mode de connaissance, alors, encore celui-ci fût-il éternel, il ne peut y avoir aucune immortalité de nature personnelle qui tienne. Par conséquent, on ne peut légitimement mettre cette conception de la connaissance et du bonheur au service d'une théodicée promettant aux individus la véritable récompense de leur vertu dans un monde à venir, comme le fait Gersonide. Examinons pourquoi il en va ainsi.

Dans notre progression vers le troisième mode de connaissance, nous nous efforçons d'acquérir des idées adéquates, de les maintenir telles et d'en augmenter le nombre. En quoi cela est-il à notre avantage ? Simplement en ce que plus nous avons d'idées, plus ce qui nous appartient demeure après la mort du corps et la fin de notre existence dans la durée, puisque les idées adéquates ne sont rien d'autre que la connaissance éternelle des choses. En d'autres termes, plus nous avons d'idées adéquates, plus le degré d'éternité de notre esprit est élevé.

P 38 : Plus l'Âme connaît de choses par le deuxième et le troisième genres de connaissance, moins elle pâtit des affects qui sont mauvais et moins elle craint la mort.

Dem : L'essence de l'Âme consiste dans la connaissance; à mesure donc que l'Âme connaît plus de choses par le deuxième et le troisième genres de connaissance, une plus grande partie d'elle-même demeure, et en conséquence une plus grande partie d'elle-même n'est pas atteinte par les affects qui sont contraires à notre nature, c'est-à-dire qui sont mauvais.

Est-on en présence d'une doctrine de l'immortalité de l'âme ${ }^{21}$ ? Dans une large mesure, il s'agit là d'une question demandant s'il y a une âme personnelle après la mort. Un esprit éternel peut-il se distinguer qualitativement d'un autre et rester relié à la vie qui était la sienne dans la durée ?

Je ne vois pas comment, pour Spinoza, un esprit éternel pourrait se distinguer qualitativement d'un autre et s'individualiser. Ou plutôt, et ce peut-être de manière plus exacte, je ne vois pas pourquoi deux esprits éternels devraient nécessairement être distincts l'un de l'autre. Ces esprits éternels ne sont composés que d'idées ou de connaissances abstraites, et il n'y a en principe rien qui les empêche d'avoir un contenu identique. Le cas limite serait celui de la connaissance parfaite, dans lequel l'esprit refléterait la compréhension totale et éternelle des choses qui est celle de Dieu : dans ce cas, un esprit éternel aurait

21. Je développe plus amplement l'idée que Spinoza niait l'immortalité de l'âme dans Spinoza's Heresy: Immortality and the Jewish Mind, Oxford, Oxford University Press, 2002, chap. 5. 
le même contenu qu'un autre. Mais même avec un degré moindre de connaissance, qu'est-ce qui empêcherait deux esprits d'avoir le même ensemble d'idées adéquates? Spinoza dit assez clairement que plus deux esprits ont des idées d'adéquates, plus ils « s'accordent l'un avec l'autre ", et que dans la mesure où nous avons des idées adéquates, nous nous " accordons » tous les uns avec les autres (IVP35).

Il est tout aussi problématique de prétendre qu'un esprit peut s'individualiser non par les éléments synchroniques qui forment son contenu, mais par son rattachement à une conscience particulière existant dans la durée lors de cette vie (ce qui, alors, lui donnerait sa dimension personnelle). Spinoza affirme assez clairement que la continuité de la mémoire est essentielle à la continuité de l'identité d'une personne dans le temps (IVP39S) ${ }^{22}$; mais il affirme également, comme le ferait tout bon cartésien, que la mémoire ne dure qu'aussi longtemps que le corps est en vie (VP21). Par conséquent, il n'y aura aucun lien à l'intérieur de la conscience entre l'esprit dans la durée et l'esprit sub specie aeternitatis.

Indépendamment de ce que l'on peut penser de ces arguments, il est vrai, bien sommaires, il y a une très bonne raison, et à notre sens la meilleure possible, pour penser que Spinoza niait l'immortalité personnelle de l'âme. Il s'agit d'une raison interne au système de Spinoza, mais qui demande que l'on prenne un peu de recul pour envisager l'ensemble de son plan philosophique, en particulier dans ses aspects moraux et religieux. Souvenons-nous que l'un des buts principaux du projet de Spinoza est de nous libérer de l'emprise des passions irrationnelles et de nous conduire vers une vie de vertu, de bonheur et de liberté - ou, du moins, vers le type d'autonomie qui nous est accessible en tant qu'agents rationnels - et que les deux passions qui l'intéressent le plus sont l'espoir et la crainte. Ce sont les passions les plus facilement manipulées par les autorités religieuses, qui cherchent à contrôler notre vie et à s'assurer de notre obéissance. Des prédicateurs sans scrupules profitent de notre tendance à la superstition en nous persuadant qu'il existe une récompense ou une punition éternelles à espérer ou à craindre après cette vie. Or, un élément essentiel de la réussite de leur discours réside dans notre conviction qu'il y a effectivement une telle vie après la mort ; que notre âme, une âme pour laquelle nous avons un attachement très intime, continuera à vivre après la mort de notre corps, et qu'il y a une immortalité personnelle. Je crois que Spinoza voulait nous délivrer d'une vie poussée aux quatre vents de l'espoir et de la crainte, c'est-à-dire d'une vie de superstition, en tuant à la racine et en éliminant la croyance fondamentale sur laquelle ces espoirs et ces craintes grandissent, savoir, la croyance en l'immortalité de l'âme. Car il se peut qu'il y ait des aspects éternels de l'esprit. Mais, nous dit Spinoza, la véritable éternité de

22. Ce n'est toutefois pas un point accordé par tous les spécialistes de Spinoza. Voir en particulier "Spinoza on the Self, Personal Identity and Immortality ", dans Spinoza: The Enduring Questions, éd. G. Hunter, Toronto, University Press of Toronto, 1996. 
l'esprit n'a rien à voir avec l'immortalité personnelle que les chefs des religions organisées nous brandissent devant les yeux en guise de motivation ou de menace.

Ce qui reste vraiment de nous après la mort, selon Spinoza, c'est un corps impersonnel de connaissances, un corps de connaissances qui, en un sens, nous appartenait déjà lors de notre vie. De la sorte, nous sommes en mesure de partager et de jouir de cette éternité dès notre existence dans la durée : ainsi, comme l'exprime Spinoza, " nous sentons et nous connaissons par expérience que nous sommes éternels ${ }^{23}$. Mais cette éternité de l'esprit n'est pas propre à nous réconforter au sens où l'on pourrait en attendre une récompense pour la vertu dans un monde à venir. Et il me semble que Spinoza voulait bien dire que Gersonide et ceux qui voulaient construire une théodicée similaire n'avaient pas le droit de prétendre que l'on devait en attendre une telle récompense.

On pourrait me reprocher d'avoir exagéré la continuité entre Spinoza et Gersonide en disant que Gersonide ne reprendrait assurément pas à son compte la négation spinoziste de l'immortalité personnelle et ses conséquences pour la théodicée. Après tout, ne soutient-il pas explicitement l'immortalité de l'âme et l'existence d'une récompense pour la vertu dans le monde à venir ? Quoique je ne sois pas entièrement convaincu que Gersonide ne désirait pas, lui aussi, rejeter la dimension personnelle de l'immortalité, il se pourrait qu'il y ait effectivement ici une discontinuité radicale entre les deux penseurs sur cette question. Mais cela ne diminue certainement aucunement l'importance du dialogue qui se joue entre Spinoza et ses précurseurs rationalistes juifs en ce qui concerne ce problème. Peut-être ne devrait-on pas voir Spinoza comme un iconoclaste qui a marqué une coupure radicale avec la pensée juive traditionnelle, mais plutôt comme un homme qui a endossé un certain courant intellectualiste du rationalisme juif et l'a simplement poussé jusqu'à ses conclusions logiques ${ }^{24}$.

23. Éthique VP23S.

24. Ce texte a été traduit de l'anglais par Syliane Charles. 